\title{
Review on Harmonics and its Eliminating Strategies in Power System
}

\author{
N. Suresh* and R. Samuel Rajesh Babu
}

\author{
Department of EIE, Sathyabama University, Chennai - 600119, Tamil Nadu, India; mailtonsuresh@gmail.com
}

\begin{abstract}
Non-linear loads are the major source of harmonics in the power system. These loads impose varying reactive-power demands that have to be compensated in order to improve the power factor and efficiently deliver the active power to the loads. This results in harmonic distortion-related problems, reducing the quality of electrical power and the performance of the power system. In this paper, the nature of harmonics that exists in the operation of various equipment, devices and components, the effects of harmonics and methods to eliminate the harmonics of power systems have been discussed briefly. The paper also reviews the pros and cons of various harmonics eliminating strategies followed in the literature and propose a new hybrid optimization method to eliminate the harmonics in multilevel inverters. The proposed hybrid optimization based harmonic elimination method calculates the switching angles for the multilevel inverter in real-time and offers a optimum switching angle at a faster convergence rate compared to other existing methods which calculate the switching angles off-line and look up tables were used, which leads to finding the near optimum switching angle and converges at a lower rate.
\end{abstract}

Keywords: Harmonics, Multilevel Inverter, Optimization Algorithm, Power System, Total Harmonic Distortion

\section{Introduction}

In the last three decades, various methodologies and novel techniques were proposed to improve the power quality. One of the parameters which affects the quality of power being harmonics, it is of great importance in the industrial field, because of the distortion, disturbance and heating effects that they cause ${ }^{1}$. In addition, there is always the chance of system resonance causing voltage and current magnification in equipment. Usually, only odd harmonics will be produced by electrical equipments, when working normally. During transient conditions, conditions of malfunction or single-phase rectification etc, even harmonics may occur ${ }^{2}$.

The advent of power electronic components such as thyristors, triacs, GTO, IGBT, power transistor, etc., have a major impact on industrial world. These components are used to develop converters which inject harmonic currents on the electrical network and draw non-sinusoidal currents from utility grids, thus contributing to degradation of power quality in distribution or industrial power systems.

This paper discusses about the sources of harmonics, the effects of harmonics and the methodologies that were proposed to eliminate or compensate harmonics in the literature for inverters in power system.

\section{Sources of Harmonics}

It is always better to discuss about the definitions of Harmonics and Total Harmonic Distortion before going for the sources of harmonics. A power supply should show a perfectly sinusoidal voltage at every residential or industrial location. But, utilities find it difficult to preserve such conditions due to various reasons. The deviation of the voltage and current waveforms from sinusoidal is said

${ }^{*}$ Author for correspondence 


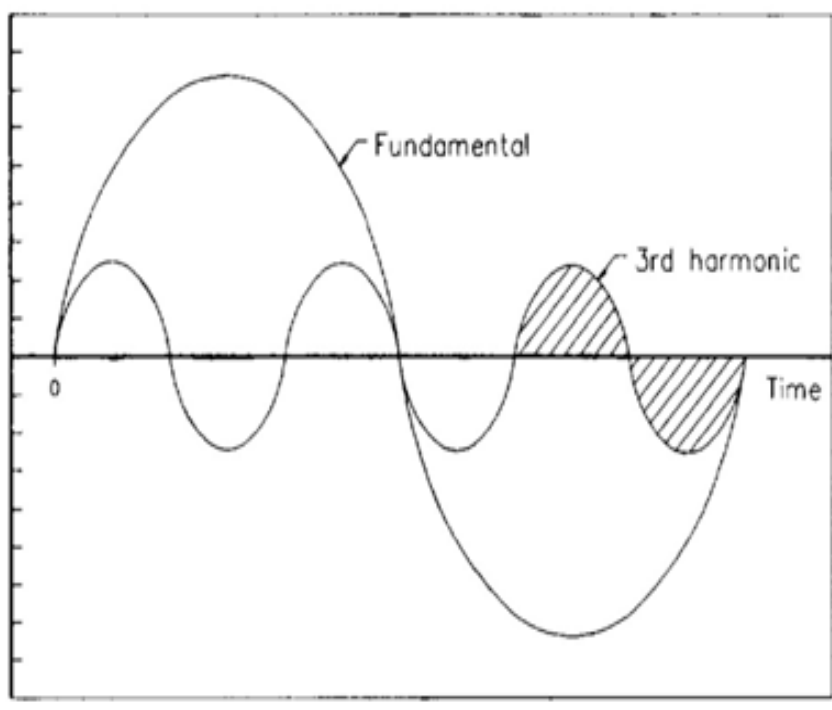

(a)

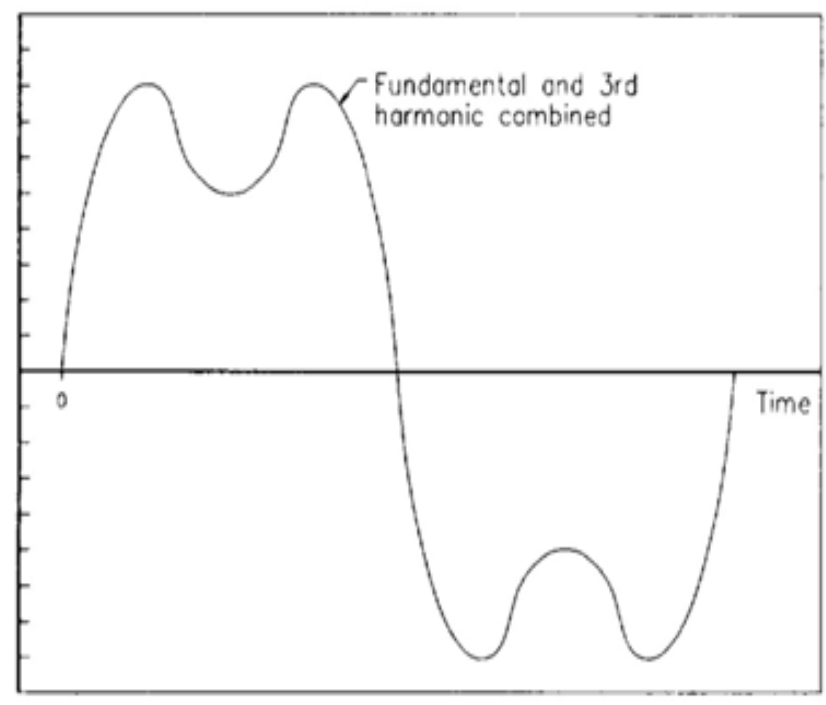

(b)

Figure 1. (a) Fundamental and third harmonic ${ }^{3}$ and (b) Fundamental and third harmonic combined ${ }^{3}$.

to be harmonic distortion. A harmonic component in a power system is defined as a sinusoidal component of a periodic waveform having a frequency that is an integral multiple of the fundamental frequency ${ }^{3}$. The fundamental and third harmonic component waveform is shown in the Figure 1.

Frequency of Harmonic Component $\mathrm{F}_{\mathrm{H}}=\mathrm{L}^{\star} \mathrm{F}$

Where,

F - fundamental frequency $\mathrm{Hz}$.

$\mathrm{L}$ - integer (order of the harmonics) (no unit).

If the frequency of the power supply is $50 \mathrm{~Hz}$, then the second harmonic frequency is $100 \mathrm{~Hz}$ and the third harmonic frequency is $150 \mathrm{~Hz}$. A distorted wave may contain harmonics of infinite order. The amplitude of the distorted wave decreases with increase in the harmonic frequency. According to IEEE 519 standard the Total Harmonic Distortion (THD) for the general system should be less than $5 \%$. The total harmonic distortion of the current is defined by the following expression,

THD $\%=\left(\mathrm{I}_{2}{ }^{2}+\mathrm{I}_{3}{ }^{2}+\ldots \ldots . .+\mathrm{I}_{\mathrm{n}}{ }^{2}\right) \wedge 0.5^{*} 100 / \mathrm{I}_{1}$

Total Harmonic Distortion (THD) can also be called as Distortion Factor (DF), Harmonic Factor (HF) etc ${ }^{12}$.

Voltage harmonics and current harmonics are produced by non-linear loads connected to the power system. Load is said to be non-linear when its impedance changes with respect to time. The sources of harmonics ${ }^{4-12}$ in power system are:

- Rectifiers (AC-DC Converters).
- Inverters (DC-AC Converters).

- Choppers(DC-DC Converters).

- Uninterrupted Power Supplies (UPS).

- $\quad$ Switched mode power supplies (SMPS).

- Fluorescent lights (CFL).

- $\quad$ Static VAR compensators.

- Variable frequency motor drives (VFD).

- Power transformer.

- Ballast inductor.

- Cycloconverters.

- Television sets.

- Personal Computers.

- Synchronous machines.

- Induction machines.

- Washing Electric arc furnaces.

- Motor-soft start units.

- Pulse-burst heating.

- Soldering equipment.

- Mercury-vapor or high-pressure sodium lamps.

- Battery Chargers.

- Refrigerators.

- Freezers.

- Machines.

- Air-conditioning devices.

- FAX machines.

- Printers.

Out of many harmonics sources listed above few of the major sources have been discussed briefly. 


\subsection{Static Var Compensators}

At the ends of long transmission lines or near sources of fluctuating power, static var compensators are used to control the voltage in power systems. Thyrsistor controlled reactor will produce about $1 \%$ of the $11^{\text {th }}$ harmonic current.

\subsection{Power Converters}

Rectifiers presents higher inductance on the dc side than on the ac side. Hence the dc current is almost constant and the converter acts as a harmonic voltage source on the $\mathrm{dc}$ side and the harmonics current source on the ac side. The harmonics produced by the pulse converters depends on the number of pulses. The harmonic component in case of pulse converters is given by the expression

$\mathrm{H}=\mathrm{LP}+/-1$

Where,

$\mathrm{H}$ - order of harmonic component (no unit).

$\mathrm{L}$ - integer (no unit).

$\mathrm{P}$ - number of pulse (no unit).

\subsection{Transformer}

Due to saturation and hysteresis characteristics, transformers in steady state will produce small level of harmonic current. High level of harmonics will be produced when transformers are energized initially, which can be as high as $60 \%$ of the rated current of the transformer.

\subsection{Rotating Machines}

Harmonic currents can be produced due to irregularities in the winding pattern or small asymmetries in the rotating machines. The resultant magneto motive force in the machine may develop harmonics. Harmonic currents were induced due to magnetic core saturations also but insignificant.

\subsection{Electric Arc Furnace}

Due to variation of arc feed material, the harmonics produced by electric arc furnaces cannot be predicted definitely. The electric arc furnace load gives worst distortion, due to the physical phenomenon of the melting with the moving electrode and molten material. The arc current waveform varies with variation in the cycle.

\section{Effects of Harmonics}

The problems caused by the harmonics on the power equipment or components ${ }^{13-17}$ are:

\subsection{Conductors}

Normally, heat will be produced in the current carrying conductors due to I2R losses. An increase in harmonic orders will produce an increase in the skin effect. As the skin effect increases more I2R losses will be produced which results in over heating of the conductors. Heating of conductors may also take place because of the proximity effect influenced by the magnetic field of harmonic currents in the adjacent conductors.

\subsection{Transformers}

Eddy current losses are directly proportional to the frequency. Hence, as the harmonic order increases there will be a increase in the eddy current losses for transformers. In addition to the skin effect, eddy current losses in transformer leads to overheating and the life of the transformer would be reduced. This increase in temperature will result in increased loss of life to the insulation of transformer which can be determined based on hot spot temperature reached by the winding.

\subsection{Capacitance}

The use of capacitors to improve the power factor has a significant influence on harmonic levels. The capacitive reactance decreases with increase in the frequency of harmonics. So because of the increased flow of current, the capacitor may get overloaded and impose higher dielectric stress. Suppose, if the resonance condition (i.e. capacitive reactance becomes equal to the inductive reactance) occurs in the circuit, then the impedance in the circuit get decreased, causing destructive damage to capacitors and other parts as well.

\subsection{Circuit Breakers and Fuses}

Low level faults in circuit breakers can be due to high degree of harmonic load current. Also, for load distortion, high di/dt ratings at zero crossings for sinusoidal waveform make the interruption difficult. Hence, harmonic load currents may lead to mal-function of circuit breakers. 


\subsection{Lights}

Life of the incandescent lamp gets decreased with the distorted power supply. If the lamp is operated at $105 \%$ rated voltage then its life gets decreases by $47 \%$. Audible noise is the problem due to harmonic currents in case of discharge lamps. In fixtures with capacitors, the capacitors together with the ballast inductor and the lamp may present a resonance problem.

\subsection{Rotating Machines}

Losses produced in the electric machines depend on the operating frequency. For an inverter producing high harmonic frequencies, core and stray losses becomes significant for induction motor. The increase in temperature in the windings causes the reduction in life of the rotating machines. Interaction between the air gap flux density and the fluxes produced by the harmonic currents in the rotor, pulsating torques are produced. Due to difference between time harmonic frequencies audible noises are produced. Other problems in rotating machines due to harmonics are equipment fatigue, bearing wear out, etc.

\subsection{Telephone Interferences}

Power system harmonics can cause greater problems than fundamental frequency because human hearing sensitivity and telephone response peak near $1 \mathrm{KHz}$. Inductive, capacitive and conductive interferences may take place between telephone line and a power line.

\section{Methods to Eliminate Harmonics}

In this section, various elimination or compensation methods for harmonics that have been handled in literature for inverters has been discussed briefly enumerating their advantages and disadvantages.

Vector-optimized harmonic elimination for single phase Pulse-With Modulated inverters ${ }^{18}$, considers dclink ripple due to finite dc-link capacitance in optimized switching angle calculation. Here pulse position is not fixed and output voltage quarter wave symmetry is not assumed. In application requiring capacitor size optimization, ripple capacitor filter can introduce lower order harmonics to flow in the ac load. These lower order harmonics are eliminated reducing the capacitor filter size. The PWM converter acts additionally as an active power filter for power factor correction. The drawback of this method is that, it does not talk about the THD factor.

In ${ }^{19}$ Fuzzy multi-objective technique integrated with Differential Evolution (DE) has been applied to optimize power factor and Total Harmonic Distortion. An attempt to increase the power factor may result in increase of THD. Such problem is solved by this approach. Multi-objective in the sense power factor and Total Harmonic Distortion has been optimized keeping selective Harmonic distortion within limits. The advantages of DE are finding global minimum regardless of initial parameter values, fast convergence and few control parameters.

Half-wave symmetry selective harmonic elimination method was implemented for five level inverter ${ }^{20}$ with varying modulation index range 0 to 1.15 . Twelve group of solutions were obtained for modulation index of 0.8 Even harmonics can be eliminated automatically in both half wave and quarter wave symmetry method. Half wave symmetry allows the variation of phase of the harmonics with the fundamental whereas only 0 or 180 degree phase shift can be provided by quarter wave symmetry. Halfwave symmetry SHEPWM can generate more solutions than quarter wave symmetrical method and asymmetry method. But complexity increases due to more number of equations.

Shuffled Frog Leaping Algorithm has been utilized $i^{21}$ to calculate switching angles for eleven level inverter. Optimal solution for the selective harmonic elimination problem was obtained at low modulation indices. The advantage of meta-heuristic SFLA includes fewer control parameters, great capability in global search and easy implementation. The disadvantage is that it is an offline method and separate lookup table is required.

Voltage harmonics in single phase inverter was reduced by composite observer method $\mathrm{in}^{22}$. To extract the in-phase and quadrature signals from periodic waveform containing harmonics composite observer was used. Here the inverter was modeled as the feedback control system with harmonics being the noise and fundamental component of periodic waveform to be the required output. Series compensation for harmonic voltage drop in the filter inductor was done by feed forward procedure. The difficulty with feed forward compensation is filter inductance, effective series resistance and delay is not precisely known. The method reduces the THD but it cannot be applied to reduce selective harmonics.

Active Power filter in phase coordinates was developed to mitigate harmonics in ${ }^{23}$. Computation of the reference 
filtering currents was performed by this method. It can handle distorted and unbalanced source voltages. Least square fitting curve method and phase-lock loop method were utilized to determine the reference currents. With the application of APC - abc THD was reduced by 18 times.

To reduce voltage distortion throughout the power system single-tuned passive harmonic filters were used $\mathrm{in}^{24}$. The harmonic voltage distortion limit and filter component limits were modeled as constraints. The size of each filter component in the power system was determined by Genetic algorithm. Equivalent resistance approach was used to place passive filters in the system. The proposed method may fail under circumstances such as resonance occurrences or insufficient capacitor buses.

A method called Notch-Filter Inserted Current Reference plus Load Current Feed-Forward Scheme (NF-CR+LCFFS) was proposed in ${ }^{25}$ to reduce Second Harmonic Current and improve the dynamic response of two stage single phase inverter. The SHC when penetrates into the front-end $\mathrm{dc}$-dc converter, it affects the conversion efficiency. The method gives superior dynamic performance under transient conditions and effectively reduces the second order harmonics. The drawback of this method is that it fails to reduce harmonics other than second order and THD parameter was not discussed.

In ${ }^{26}$ Optimal Pulse Width Modulation based on harmonic injection and equal area criteria has been applied for Selective Harmonic Elimination in multilevel converters with unbalanced dc voltage source. Only four equations are needed in this method, irrespective of the number of voltage level. The harmonics in the sine wave can be cancelled by injecting selective negative harmonics because of equal area criteria. The advantage of this method is that solving higher order non-linear equations are not necessary, so advanced algorithms are not required. In other methods, the number of equation grows non-linearly with switching angles whereas here number of equations increases linearly. The disadvantage is that it uses lookup table to calculate switching angles.

Harmonic reduction in current source converter using fuzzy logic was implemented $\mathrm{in}^{27}$. Here, Space Vector Modulation technique generates lower order harmonics in the CSC ac-side current when operated under low switching frequencies. Fuzzy logic technique is used to determine the state ON time of the CSC-SVM to minimize the lower order harmonics. The merit of this method are lower order harmonics and Harmonic Distortion
Factor are reduced to a great extent, but not suitable for higher order harmonic elimination. Lookup table is used.

Real-time implementation of Selective Harmonic Elimination Pulse Width Modulation in single phase inverter using Generalized Hopfield Neural Network was designed $\mathrm{in}^{28}$. The problem of solving the non-linear transcendental equations to find the switching patterns for the inverter is redrafted as the optimization problem and it is solved by using GHNN. Energy function of Ordinary Differential Equation for the GHNN was solved by RungeKutta method. The method eliminates $5^{\text {th }}, 7^{\text {th }}, 11^{\text {th }}$ and $13^{\text {th }}$ order harmonics while retaining the fundamental component. The method was simulated using MATLAB and hardware implementation is done using ARM processor MOSFET acted as a switching device. On-line calculation of switching angle was done in this method.

Particle Swarm Optimization (PSO) algorithm has been utilized in ${ }^{29}$ to selectively eliminate harmonics of 11-level $\mathrm{H}$-bridge inverter with unequal dc source voltages. The method eliminates the specific harmonics with lower total harmonic distortion. This method solves the asymmetry of the transcendental equations. The calculation of each set of solution takes 029s. In hardware implementation FPGA produced gating signals for the MOSFET switching device. The probability of optimal finding of the switching angles decreases with increase in the number of switching which can be due to increase in the harmonic order.

Asymmetrically defined five-level Multilevel Selective Harmonic Elimination Pulse Width Modulation (MSHEPWM) method was proposed in $^{30}$. The switching angles were calculated using biased search optimization algorithm. In the standard modulation region, the method offers higher bandwidth, whereas in the .over modulation region, higher amplitude of the fundamental component is possible with reduced bandwidth. The PWM signals are generated by means of Digital Signal Processor (DSP) to control the IGBT (Insulated Gate Bipolar Transistor) of H-bridge inverter. The method was found superior to MPS-SPWM (Multi-carrier Phase Shifted Sinusoidal Pulse Width Modulation) in terms of converter bandwidth and modulation index. Compared to half-wave symmetry and quarter wave symmetry, different voltage levels can be generated by the asymmetrical multilevel inverter. Size of the output filter required for the method is less.

To reduce harmonic distortions in power systems cascaded active power filter were used ${ }^{31}$. Two active power 
filters were connected in cascade with different operating frequencies and different power ratings. In power systems, the amplitude of harmonics and harmonic orders are inversely proportional to each other. Similarly, switching frequency of devices is inversely proportional to power capacity. So, one of the active power filters with low power rating and high switching frequency compensates for higher order harmonics and the other active filter with high power rating and low switching frequency reduces the lower order harmonics. Thus the cascaded active filter reduces higher harmonics as well as the lower order harmonics effectively. The demerits of the active harmonic elimination is that to reduce higher order harmonics high switching frequency is needed and also to generate firing signals for the switches control procedures becomes complex.

Hybrid Real Coded Genetic Algorithm (HRCGA) is utilized in ${ }^{32}$ to find the optimal solution of selective harmonic elimination pulse width modulation for cascaded multilevel inverter. Considering both equal and non-equal dc sources. HRCGA reduces the computation burden of the non-linear transcendental equations. The method was implemented and tested for five levels and seven level inverter with multiple sets of solution. IGBT and digital signal processor were used for hardware implementation. Higher converter bandwidth and multiple solutions were the advantages of this method. For more number of harmonics to be reduced, complexity of the cost function increases leading to increase in the number of iterations. The cascaded inverter is shown in the Figure 2.

Reduction of harmonics in inverters was done by Walsh waveform analytic technique in $^{33}$ Linear algebraic equations were solved instead of non-linear transcendental equations to get optimized switching angles. The switching angles and the fundamental amplitude was related by straight-line curve fitting method. Both unipolar switching scheme and bipolar switching scheme can be applied for the Walsh function algorithm. Unipolar switching scheme was found superior to bipolar switching scheme in terms of harmonic loss factor and second order distortion factor. The advantage of the method is that switching angles was calculated online and no lookup table was used.

Multiple solutions for selective harmonic elimination for three phase Pulse Width Modulated Inverters were obtained by Homotopy method and mathematical induction algorithm in ${ }^{34}$ For the given degree of freedom $\mathrm{M}$ solutions are obtained for constant voltage harmonic

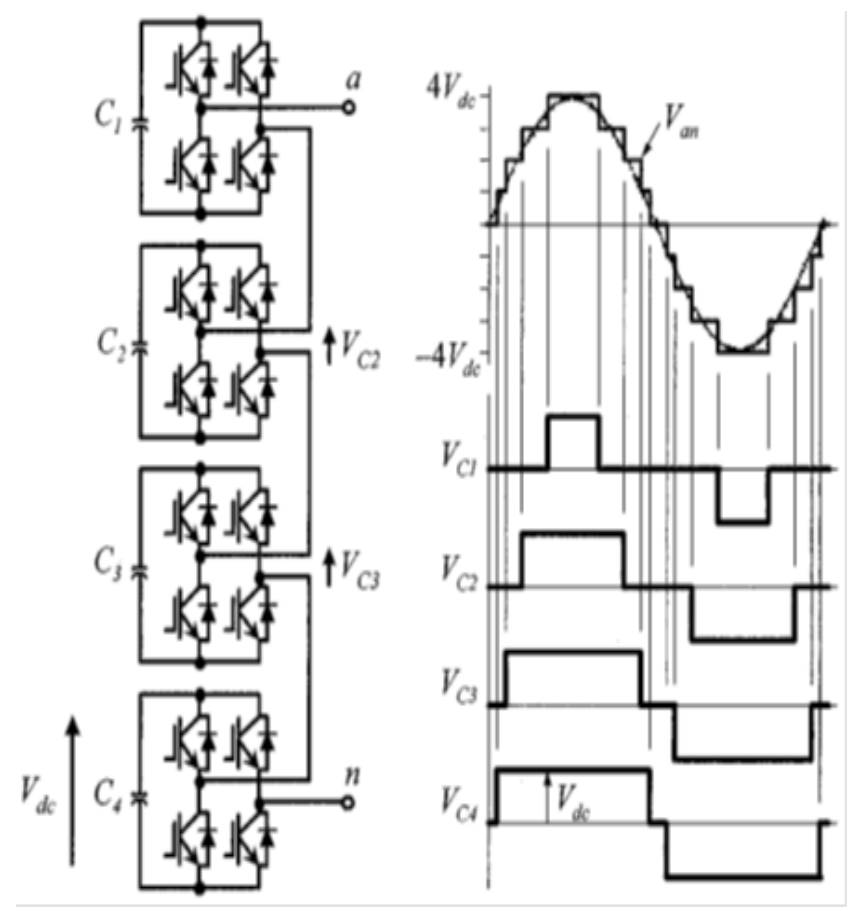

Figure 2. Cascaded inverter topology and its associated waveform ${ }^{41}$.

elimination method and variable voltage harmonic elimination method. By CV method $\mathrm{M}$ harmonics were eliminated whereas by the VV method M-1 harmonics were reduced. The homotopy method effectively reduces the lower order harmonics but it did not give any information about total harmonic distortion.

Active harmonic elimination method was proposed $\mathrm{in}^{35}$. to eliminate lower order as well as higher order harmonics of 11-level cascaded H-bridge converter. Initially, resultant theory was applied to reduce lower order harmonics. Then, the higher order harmonics are found out and subtracted from original voltage waveform to reduce them. Field Programmable Gate Array (FPGA) was used for hardware implementation. The method was tested for both equal dc voltages and unequal dc voltages and found to have very low total harmonic distortion. The switching frequency need to be higher to reduce more harmonics of the multilevel inverter. In the resultant method, the lower order harmonic elimination may introduce higher order harmonics. Also, it can determine solution only in the certain modulation indices. When the level or dc input voltage is altered, resultant method becomes complex since, it needs more expressions.

Bee algorithm has been applied to 7-level inverter to eliminate lower order harmonics by selective harmonic 
elimination pulse width modulation in $^{36}$. Simulation results with MATLAB showed the superior performance of Bee algorithm over Genetic algorithm based on global minima and higher convergence rate. The backlog of Bee algorithm is that the code is complex and the runtime is more compared to genetic algorithm. For BA switching angles are calculated offline and the lookup table was stored in AVR microcontroller. Experimental results show that the solutions near probability to attain global minima for many run is more in BA compared to GA. To get exact solution, population size need to be increased which adds to the complexity and the duration of solving the problem is more for both Bee algorithm and Genetic algorithm.

In 7-level diode clamped inverter, harmonics are eliminated by means of radial basis function neural network method ${ }^{37}$. The proposed method solves the harmonic elimination problem with ease in implementation. Faster convergence rate and accuracy are the key features of the radial basis function neural network method. A well trained artificial neural network has the inherent capability of generating the solution. Real time control can be realized as it is possible to determine the switching angles quickly. Simulation was done by using SIMULINK of MATLAB considering modulation index of 0.78 . The results show that this method was satisfactory to eliminate lower order harmonics only in specific modulation region.

Based on the foraging behavior of a colony of ants, a novel algorithm for selective harmonic elimination in pulse width modulation was developed ${ }^{38}$. Here the ant colony optimization algorithm for reducing harmonics in inverters was compared with Newton-Raphson method and Genetic Algorithm. The method offers guaranteed near optimum convergence with random initial guess. The convergence rate of the algorithm was improved by the introduction of step length of the movement of ants. Order of the harmonics eliminated and the THD information were not given. The runtime for ACS is $6.7 \mathrm{~s}$ whereas for GA it is $12.5 \mathrm{~s}$. Hardware implementation was done using IGBT switches and 8085 microprocessor.

Quarter wave symmetrical method for selective harmonic elimination pulse width modulated 5-level inverter was proposed ${ }^{39}$. The method was based on equal area criteria and superposition of center of gravity. With the modulation index $\mathrm{M}$ varying from 0 to 1.15 , harmonic orders from $5^{\text {th }}$ to $35^{\text {th }}$ order were eliminated by this method Simulation was done by Psim $^{6}$ package. Quarter wave symmetry SHEPWM has lesser number of switching angles compared to half-wave symmetrical method which makes the computation to be simple. Broader solution space with wide range of modulation index was the key feature of this method. But the number of solutions provided by quarter wave symmetrical method is less compared to the half wave symmetrical method.

Harmonic elimination and optimization of the stepped voltage of a 13-leve inverter was made by Bacterial Foraging Algorithm (BFA $)^{40}$. Comparison between bacterial foraging and genetic algorithm was done by simulating in MATLAB software. Simulation results show the superiority of BFA over GA in terms of running time and probability of convergence to a global minima. The method significantly eliminates the lower order harmonics retaining the fundamental component.

\section{Proposed Method}

The various harmonic eliminating or compensation strategies adopted in the last three decades have been reviewed. Based on this survey a new methodology to control harmonic distortion in multilevel inverter is introduced. In the proposed method Selective Harmonic Elimination Pulse Width Modulation technique to reduce harmonics is implemented by means of Particle Swarm Optimization-Back propagation Algorithm. The PSOBPA method calculates the switching angles required for the inverter on-line. So no separate look-up table is required. The key advantages of the proposed optimization method to minimize harmonic distortion are: 1 . Faster convergence rate, 2. Convergence rate and accuracy at global optima is higher, 3 . More chance of escaping from local minima and 4. Real-time solving of harmonic elimination equations.

\section{Conclusion}

Sources of harmonic distortions that can occur in the non-linear loads of power system and the associated problems were discussed briefly. Complete review of various methodologies to eliminate or compensate harmonics for inverters that were followed in the literature was done. Based on this review a new hybrid optimistic method called PSO-BPA to eliminate harmonics by calculating switching angles on-line for Selective Harmonic 
elimination Pulse Width Modulation Technique of multilevel inverter is introduced in this paper.

\section{References}

1. Barnes R, Balasingham D. Public supply system disturbances caused by rail traction loads. IEEE Symposium on Urban Railways; 1980.

2. Barnes R. Harmonics in power systems. Power Engineering Journal. 1989; 11-5.

3. Henderson RD, Rose PJ. Harmonics: The effects on power quality and transformers. IEEE Transactions on Industry Applications. 1994; 30:528-32.

4. Yacamini R. Power system harmonics Part 1 Harmonic sources. Power Engineering Journal. 1994; 193-8.

5. Francisco C, De La Rosa. Harmonics and Power Systems. Taylor and Francis Group LLC; 2006.

6. Shipp D, et al. Expert system for analysis of electric power system harmonics. IEEE Industry Application Magazine; 1995. p. 34-9.

7. Pomilio JA, Deckmann SM. Characterization and compensation of harmonics and reactive power of residential and commercial loads. IEEE Transactions on Power Delivery. 2007; 22:1049-55.

8. IEEE Working Group on Power System Harmonics. Power System Harmonics: An Overview. IEEE Transactions on Power Apparatus and Systems. 1983; 102:2455-60.

9. Samuel Rajesh Babu R, Henry J. Soft switched isolated ZVT boost DC-DC converter with coupled inductors. Journal of Electrical Engineering. 2012; 1-10.

10. Samuel Rajesh Babu R, Henry J. A comparative analysis of DC-DC converters for renewable energy systems. Proceedings of the International Multiconference of Engineers and computer scientist; Malaysia. 2012.

11. Stratford RP. Rectifier harmonics in power systems. IEEE Transactions on Industry Applications. 1980; 16:271-6.

12. Thomas S, Lai KJ-S. Costs and benefits of harmonic current reduction for switch-mode power supplies in a commercial office building. IEEE Transactions on Industry Applications. 1996; 32:1017-25.

13. Coleman W, Smith Jr. Power systems and harmonic factors Causes, effects and remedies. IEEE Potentials. 2002; 10-2.

14. Wagner VE, et al. Effects of harmonics on equipment. IEEE Transactions on Power Delivery. 1993; 8:672-80.

15. Elmoudi A, et al. Effect of harmonics on transformers loss of life conference record. IEEE International Symposium on Electrical Insulation. 2006; 408-11.

16. Joseph S, Subiak Jr, Mcquilkin JS. Harmonics - causes, effects, measurements, and analysis: An update. IEEE Transactions on Industry Applications. 1990; 26:1034-42.
17. IEEE Working Group on Power System Harmonics. Power Line Harmonic Effects on Communication Line Interference. IEEE Power Engineering Review. 1985; 257887.

18. Hadji S, et al. Vector-optimized harmonic elimination for single-phase pulse-width modulation inverters/converters. IET Electric Power Applications. 2007; 1:423-32.

19. Darvishi A, et al. Fuzzy multi-objective technique integrated with differential evolution method to optimise power factor and total harmonic distortion. IET Generation, Transmission and Distribution. 2011; 5:921-9.

20. Fei W, et al. Half-wave symmetry selective harmonic elimination method for multilevel voltage source inverters. IET Power Electron. 2010; 4:342-51.

21. Huibo Lou, et al. Fundamental modulation strategy with selective harmonic elimination for multilevel inverters. IET Power Electron. 2014; 7:2173-81.

22. Selvajyothi K, Janakiraman PA. Reduction of voltage harmonics in single phase inverters using composite observers. IEEE Transactions on Power Delivery. 2010; 25:1045-57.

23. Medina-Rios A, Ramos-Carranza H A. An active power filter in phase coordinates for harmonic mitigation. IEEE Transactions on Power Delivery. 2007; 22:1991-3.

24. Chang GW, et al. A new method of passive harmonic filter planning for controlling voltage distortion in a power system. IEEE Transactions on Power Delivery. 2006; 21: 305-12.

25. Zhu G, et al. On the reduction of second harmonic current and improvement of dynamic response for two-stage single-phase inverter. IEEE Transactions on Power Electronics. 2015; 30:1028-41.

26. Ahmadi D, et al. A universal selective harmonic elimination method for high-power inverters. IEEE Transactions on Power Electronics. 2011; 26:2743-52.

27. Naguib MF, Lopes LAC. Harmonics reduction in current source converters using fuzzy logic. IEEE Transactions on Power Electronics. 2010; 25:158-67.

28. Balasubramonian M, Rajamani V. Design and real time implementation of SHE PWM in single phase inverter using generalized hopfield neural network. IEEE Transactions on Industrial Electronics. 2013; 1-11.

29. Taghizadeh H, Tarafdarhagh M. Harmonic elimination of cascade multilevel inverters with non equal DC sources using Particle Swarm Optimization. IEEE Transactions on Industrial Electronics. 2010; 57:3678-84.

30. Agelidis VG, et al. A five-level symmetrically defined selective harmonic elimination PWM strategy: Analysis and experimental validation. IEEE Transactions on Power Electronics. 2008; 23:19-26.

31. Huang SJ, Wu JC. Design and operation of cascaded active power filters for the reduction of harmonic distor- 
tions in a power system. IEEE Proceedings of Generation, Transmission and Distribution; 1999. p. 193-9.

32. Dahidah MSA, Agelidis VG. Selective harmonic elimination PWM control for cascaded multilevel voltage source converters: A generalized formula. IEEE Transactions on Power Electronics. 2008; 23:1620-30.

33. Liang T-J, et al. Inverter harmonic reduction using walsh function harmonic elimination method. IEEE Transactions on Power Electronics. 1997; 12:971-82.

34. Kato T. Sequential homotopy-based computation of multiple solutions for selected harmonic elimination in PWM inverters. IEEE Transactions on Circuits and Systems - I: Fundamental Theory and Applications; 1999. p. 586-593.

35. Du Z, et al. Active harmonic elimination for multilevel converters. IEEE Transactions on Power Electronics. 2006; 21:459-69.

36. Kavousi A, et al. Application of the bee algorithm for selective harmonic elimination strategy in multilevel inverters.
IEEE Transactions on Power Electronics. 2012; 27:1689-96.

37. Reza Banaei M, Shayan PA. Solution for selective harmonic optimization in diode-clamped inverters using radial basis function neural networks. IET Power Electron. 2014; 7:1797-804.

38. Kinattingal S, et al. Inverter harmonic elimination through a colony of continuously exploring ants. IEEE Transactions on Industrial Electronics. 2007; 54:2558-65.

39. Fei W, et al. A generalized formulation of quarter-wave symmetry SHE-PWM problems for multilevel inverters. IEEE Transactions on Power Electronics. 2009; 24:1758-66.

40. Reza-Salehi, et al. Harmonic elimination and optimization of stepped voltage of multilevel inverter by bacterial foraging algorithm. Journal of Electrical Engineering and Technology. 2010; 5:545-51.

41. Rodriguez J, et al. Multilevel inverters: A survey of topologies, controls, and applications. IEEE Transactions on Industrial Electronics. 2002; 49:724-38. 